\title{
Captures
}

Figures, théories et pratiques de l'imaginaire

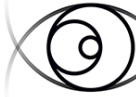

\section{Marie Darrieussecq et Christine Angot, récidivistes du réel}

C A P P Figures, théories et pratiques de limaginaire evve interdisciplinaire

\section{Eftihia Mihelakis}

Volume 4, Number 1, May 2019

Paroles diffamantes, images infamantes

URI: https://id.erudit.org/iderudit/1060153ar

DOI: https://doi.org/10.7202/1060153ar

See table of contents

Publisher(s)

Figura, Centre de recherche sur le texte et l'imaginaire

ISSN

2371-1930 (digital)

Explore this journal

Cite this article

Mihelakis, E. (2019). Marie Darrieussecq et Christine Angot, récidivistes du réel. Captures, 4(1). https://doi.org/10.7202/1060153ar

\section{Article abstract}

Si la notion d'infamie intéresse aujourd'hui le système juridique et médiatique, elle n'en concerne pas moins les études littéraires dans lesquelles il semblerait qu'elle ait un sens alternatif, plus proche de celui qu'en donne Michel Foucault. L'écriture de Marie Darrieussecq et Christine Angot peut être lue comme un geste littéraire qui nomme et montre l'en-deçà de la réalité, ces restes qui hantent l'ordre du discours.
Tous droits réservés (C) Eftihia Mihelakis, 2019

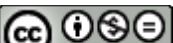

This document is protected by copyright law. Use of the services of Erudit (including reproduction) is subject to its terms and conditions, which can be viewed online.

https://apropos.erudit.org/en/users/policy-on-use/ 
Version enrichie de cet article : http://revuecaptures.org/node/3638

\section{Marie Darrieussecq et Christine Angot, récidivistes du réel}

\section{Eftihia Mihelakis}

Résumé :

Si la notion d'infamie intéresse aujourd'hui le système juridique et médiatique, elle n'en concerne pas moins les études littéraires dans lesquelles il semblerait qu'elle ait un sens alternatif, plus proche de celui qu'en donne Michel Foucault. L'écriture de Marie Darrieussecq et Christine Angot peut être lue comme un geste littéraire qui nomme et montre l'en-deçà de la réalité, ces restes qui hantent l'ordre du discours.

If the notion of infamy is of interest today to the legal and media systems, it also concerns literary studies in which it seems that it can take on an alternative meaning, closer to that given by Michel Foucault. This article reads Marie Darrieussecq and Christine Angot's literary works as a gesture that names and shows this "minor" reality, its "remains," which haunt the order of discourse.

Stigmatisés, étiquetés, marqués viles, honteux, répugnants, lâches, ou calomnieux, les écrivains infâmes sont reconnus coupables d'une offense sans qu'il y ait eu crime stricto sensu. II faut seulement qu'il y ait eu ternissement public de la réputation d'une personne. Mais le mot « infamie » prend un sens alternatif dans «La vie des hommes infâmes » de Michel Foucault (1977). Foucault explique comment l'infamie concerne les gens invisibilisés plutôt que ceux qui sont illustres ou célèbres. L'infamie, écrit-il, est une aura qui enveloppe l'accusé de silence. Ces « pauvres esprits » (Foucault, 1977: 16), « un moine scandaleux ou un usurier fantasque et inconséquent » (14), voilà quelques exemples d'antan, rangés dans les annales apocryphes de l'infamie, là où des « milliards d'existences [...] sont destinées à passer sans traces » (16). Par ailleurs, comme le souligne Mario Colucci à propos de l'essai de Foucault, loin d'être reconnus pour leur célébrité ou élevés sur le piédestal de la reconnaissance pour leurs contributions remarquables, les hommes ou les femmes infâmes appartiennent « au gris et à l'ordinaire » (2005: 144), à l'anonymat.

Plusieurs critiques littéraires, à l'instar de Jacques Dubois, insistent sur le fait que « Christine Angot est une romancière bien intégrée au champ littéraire, y occupant une place en vue après la publication d'une dizaine de romans et de textes » (2011: §8). Le même constat peut être fait à propos de Marie Darrieussecq, dont l'œuvre est souvent comparée ou étudiée en tandem avec celle d'Angot 1 . Aurait-on donc tort d'étiqueter d'infâmes Marie Darrieussecq ou Christine Angot, traînées en justice ou obligées de comparaître devant le tribunal de l'opinion publique? Avec chaque livre, Angot et Darrieussecq semblent commettre une nouvelle infraction. Que cette dernière soit passible d'une condamnation pénale ou publique, qu'elle soit considérée un crime, un délit ou une calomnie, elles récidivent. Les accusations parsèment certes l'imaginaire contemporain, mais avec quel genre de discours ces auteures se préoccupent-t-elles réellement des griefs symboliques, psychiques, 
matériels?

Dans le cadre du présent article, parler du geste de récidive chez Darrieussecq comme chez Angot, c'est montrer comment l'écriture hante l'ordre du discours. Nous nous inspirons cependant de l'étude de Foucault sur l'infamie pour poser l'hypothèse que la littérature n'est pas un simple contre-discours au juridique, son altérité radicale. Le matériau discursif de l'infamie est composé de « fragments $d[u]$ discours traînant les fragments d'une réalité dont ils font partie » (Foucault: 15). Nous espérons ainsi rendre visible un visage peu connu de l'infamie : autant les petites traces de la réalité qui hantent le social que l'« écriture de l'infamie » (Demartini, 2002: 129), le cadre spécifique de ses mécanismes littéraires, c'est-à-dire, la logique même de son fonctionnement.

\section{Les avatars de la diffamation}

Nous assisterions en France depuis les années 2000 à des accusations de tout ordre, autant légales que d'ordre moral ou éthique, intentées contre des écrivains, si bien qu'avec cette multiplication semble aussi régner un nuage de confusion quant à la compréhension des termes utilisés pour les accuser. On n'est pas étonné du propos émis par certains critiques, dont ici celui de Christian Ruby : "Campagnes de protestations, réclamations pour atteinte à tel ou tel droit, tentatives de censure sur tel ou tel écrit ou sur telle ou telle chanson, les processus punitifs relatifs à des œuvres d'art ne cessent de s'amplifier depuis quelques années. 》 (2011.) À en croire le récent article de David Caviglioli paru dans Le Nouvel observateur, « [s]i on suit la logique des condamnations récentes, les ruptures amoureuses, la vie familiale, les turpitudes des personnages publics sont des territoires désormais interdits à toute forme de littérature » (2013). Si, comme le remarque Ania Wroblewski, en faisant référence aux travaux d'Agnès Tricoire (2011) et Gisèle Sapiro (2011), les études démontrent que « le rapport non seulement entre la création et la loi mais aussi celui entre la création et la vie privée est aujourd'hui plus tendu que jamais » (2016: 21), nous serions tout aussi bien avisés d'explorer des horizons interprétatifs à travers lesquels nous pourrions voir par-delà la distinction qui opposerait le discours juridique et médiatique, d'une part, et le discours littéraire de l'autre (Compagnon, 2017). Avant de développer adéquatement l'enjeu de notre étude, il apparaît primordial de préciser clairement les dimensions opérationnelles qui composent l'histoire de la diffamation en France.

Dans l'histoire de la littérature française moderne et contemporaine (depuis la fin du XIX'e siècle jusqu'à aujourd'hui), le domaine littéraire et artistique est plus ou moins systématiquement policé par le domaine juridique, et ce, autour d'une idée spécifique de la diffamation. Dépliant la problématique « De la littérature comme sport de combat » dans le cadre du séminaire Littérature française moderne et contemporaine. Histoire, critique, théorie donné au collège de France en 2016-2017, Antoine Compagnon fait bien d'insister sur la longue histoire de la diffamation à travers laquelle le littéraire (la presse) s'oppose au juridique. Compagnon 
présente ce lien autour de la figure du duel (de la confrontation violente par la parole ou par les actes) dans le cours du 7 mars 2017, montrant ainsi que la littérature a causé de vrais dommages. L'objectif de notre présente réflexion n'est pas de répéter le remarquable travail d'Antoine Compagnon sur l'histoire de la guerre littéraire. II est néanmoins opportun de rappeler l'essence de ses propos tenus spécifiquement lors de la séance « Tropes de la guerre littéraire. Diffamation/duel » du 7 mars 2017 autour de l'article 29 de la loi du 29 juillet 1881 sur la liberté de la presse. Votée sous la III ${ }^{\mathbb{E}}$ République, cette loi définit et encadre encore aujourd'hui les libertés et les responsabilités de la presse française (au sens large, ce qui inclut la littérature). Cette loi impose un cadre légal et éventuellement pénal à la publication pour protéger les personnes de l'atteinte à la vie privée $\underline{2}$. Selon cette loi, « [l]a diffamation [...] n'implique pas nécessairement la fausseté des faits imputés ou allégués, elle dénote seulement d'une part l'intention de nuire, de l'autre le dommage causé » (Roux, 1882: 136). Conformément à cette définition, la diffamation et l'injure sont des délits (le terme calomnie ne serait plus souscrit à la loi). Jusqu'en 2006, il était impossible d'apporter une preuve pour se blanchir contre une accusation de diffamation. Mais depuis le jugement de la $17^{e}$ chambre du Tribunal de grande instance de Paris, aussi appelée Chambre de la presse, datant du 17 mars 2006, dans une affaire opposant la mairie de Puteaux à Christophe Grébert, animateur du site monputeaux.com :

Si les imputations diffamatoires sont réputées faites dans l'intention de nuire, le prévenu peut cependant justifier de sa bonne foi et doit, à cette fin, établir qu'il poursuivait, en diffusant les propos incriminés, un but légitime exclusif de toute animosité personnelle, qu'il a conservé dans l'expression une suffisante prudence et qu'il avait en sa possession des éléments lui permettant de s'exprimer comme il l'a fait. (Wikipédia, [s. d.].)

Dans tous les cas, la diffamation, qu'elle soit publique, privée ou discriminatoire, appartient toujours à la catégorie légale des délits contre la personne. Si la diffamation répond à un signal de blessure physique ou psychique dont les intentions peuvent désormais être prouvées ou discréditées, elle semble aussi entretenir une corrélation avec les attentats à la pudeur, dont l'histoire s'inscrit selon Bastien Fanthou « dans un genre précis; la victime est toujours (ou presque) une fille, et l'agresseur, un homme » (2008: 11). C'est ici que la diffamation ressemble à l'acte infamant, car l'infamant est ce qui « flétrit l'honneur, la réputation » (Robert: 1323) d'une victime au sein d'un rapport de pouvoir hiérarchique.

Contrairement à la diffamation ou à l'acte infamant, lesquels peuvent avoir des similarités avec l'attentat à la pudeur, l'infamie concerne de façon générale l'objet du dégoût. Est infâme, ce qui est « bas et vil » (Robert: 1323). Est infâme, écrit Mary Douglas, ce qui déroge aux règles du tabou : «l'existence des tabous n'est plus une énigme incompréhensible mais la manifestation du souci intelligible de protéger la société contre les comportements qui pourraient la mettre en danger. » (2001 [1967]: 191.) II importe de rappeler, à la suite de 
Julia Pecker, que « tout ce qui porte atteinte à l'intégrité physique des corps entre en résonance avec l'immonde » (2010: 65) et que ce qui est immonde « a un caractère d'impureté morale » (Robert: 1281). L'infâme est le signal du dégout. II crée un effet de répulsion. Pour Douglas, « certaines pollutions servent d'analogies pour exprimer une idée générale de l'ordre social » (2001: 25). On traite quelque chose de souillé comme on dénonce une figure ou une idée considérée dysfonctionnelle pour l'hygiène de la cité. La dénonciation est la part visible d'un registre qui détient des modalités du caché (la souillure), la première servant à renforcer la légitimité des règlements (de l'ordre social) dont le but est d'éliminer le caractère visible du tabou.

Plus qu'un simple objet de repoussoir, l'objet du tabou, la personne infâme, révèle la part du discours qui doit demeurer cachée. Or, l'objet de l'infamie ne peut pas être cantonné dans le rôle de victime comme dans le cas de ce qui est infamant. Le paradoxe du tabou existe dans le fait que ce qui est scandaleux, infâme — la part du discours qui est cachée — demande sans cesse à être révélé. Par exemple, après avoir publié Tom est mort (2007), Marie Darrieussecq se sent obligée de produire « un certificat doloriste » (2010: 17) non pas pour se protéger contre une possible accusation de diffamation, mais parce que son roman a jeté la lumière sur l'« une des idéologies dominantes » (134) du XXI e siècle, soit celle qui privilégie « le droit des victimes [...] [où] on est d'autant plus légitime qu'on souffre d'une injustice » (134). Ce qui est ressorti ici est le renforcement des protocoles de surveillance qui soutiennent une hiérarchie des souffrances : puisque la mort d'un enfant marquerait un des « tabous majeurs » (Otchakovsky-Laurens, 2007) de nos sociétés, on n'aurait pas le droit de la raconter sans l'avoir vécue de proche. Mais qu'est-ce qui détermine la distance acceptable du proche? Estce que c'est la qualité de la distance qui détermine l'intensité du scandale?

\section{De troublantes séries autofictionnelles}

Jamais formellement accusée par une cour juridique, l'écrivaine Marie Darrieussecq fait l'objet de plaintes quant à l'authenticité de ces écrits. "Mon problème c'est celui de la calomnie » (2010: 370), écrit-elle. La calomnie, comme on l'a vu plus haut, n'est plus régie par la loi juridique, mais par le procès médiatique ou le tribunal de l'opinion publique. À la parution de son livre Naissance de fantômes (1998), Marie Ndiaye — qui n'a pas porté plainte - reproche à Darrieussecq $\underline{3}$ de s'être inspirée de ses deux romans, Un temps de saison (1994) et La Sorcière (1996), dans lesquels il est ouvertement question de la désagrégation familiale. Ayant fait scandale dans la scène médiatique française, la polémique Laurens-Darrieussecq a aussi commencé avec une condamnation publique (Laurens, 2007) autour de la question des intentions face au

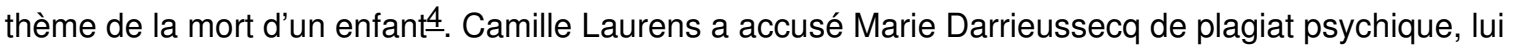
reprochant d'avoir piraté son récit autobiographique, Philippe (1995), pour écrire un roman, Tom est mort (Strasser, 2012). À l'automne 2007, « Laurens a dénié le droit à Marie Darrieussecq d'avoir raconté la mort 
d'un enfant alors qu'elle ne l'avait pas elle-même vécue » (Rérolle, 2010). Toutefois, l'accusation n'est pas considérée viable et aucune poursuite n'est retenue contre Darrieussecq. Laurens s'est même vue remerciée par P.O.L., son éditeur, pour ses propos considérés diffamatoires dans l'opinion publique.

Aussi scandaleux qu'ait pu être le geste d'écrire sur le deuil fictif d'un enfant mort, lui aussi fictif, l'on sait que Marie Darrieussecq n'a pas été renvoyée de la cité des lettres. Elle a multiplié les prises de parole. Dans Rapport de police. Accusations de plagiat et autres modes de surveillance de la fiction(2010), un essai alliant recherche psychanalytique et enquête journalistique, Darrieussecq cherche à comprendre pourquoi la société se cache derrière le masque de l'accusation. Rérolle emprunte la métaphore du fusil pour décrire le geste d'écriture des écrivaines accusées d'infamie. Reprenant cette idée de l'assassinat de l'originalité développée par Jess-Cooke et Verevis (2010: 5), l'on peut stipuler que l'auteure fait de son écriture un geste qui partirait à l'assaut. Darrieussecq ne range pas son fusil une fois qu'elle l'a chargé (avec Tom est mort, par exemple). Elle décide de « change[r] son fusil d'épaule » (Rérolle, 2010) pour réécrire (Rapport de police, par exemple). Le terme balistique (le « fusil ») est donc choisi à dessein.

Ce n'est pas le thème de l'enfant mort qu'elle insisterait à mettre à nouveau de l'avant dansRapport de police. Dans un entretien avec Nelly Kaprièlian, Darrieussecq précise : "Tous mes livres enchâssent un enfant mort, c'est-à-dire qu'ils sont comme des coffrets — ou des châsses, au sens médiéval — autour d'un enfant mort. " (Kaprièlan, 2010: 18.) C'est la raison pour laquelle l'auteure compare ce travail d'enchâssement à un « geste littéraire » (Kaprièlan: 4). Au lieu de retravailler un thème, elle s'applique à « retourner les clichés comme des pierres pour voir ce qui remue dessous » (Kaprièlian: [np]). Le geste littéraire expose à la lumière les clichés, ces lieux communs, ces banalités que l'on répète en termes identiques dans l'ordre du discours. Seulement, les livres sont aussi « des miroirs » où chacun « se voit à chaque page » (Darrieussecq, 2010: 32). Si l'écriture n'a pas de point originel, cela n'empêche pas qu'elle soit vécue comme une apostrophe. Comme l'écrit Francesca Forcolin, « la société considère cette écriture offensive et amorale puisqu'elle dévoile le plus intime, [alors qu'elle est] habituée à tourner le regard de l'indicible, du non-dit, de l'horreur et de la violence souvent cachée » (2011: 57). Les guerres littéraires contre Darrieussecq ont prouvé que celui qui tire est tout autant celui sur qui l'on tire.

Darrieussecq se retrouve « à préciser que les fantômes [...] n'ont d'existence que fictive » (Darrieussecq, 2010: 17). Cette puissance de l'imagination, le fait « qu'écrire invente des mondes, voilà ce qui heurte à l'incrédulité [...], voilà qui stupéfie » (Darrieussecq, 2010: 18). La littérature ne ferait que traîner un mal irrémédiable de la vie à la littérature sans que l'origine ne soit identifiable, alors que le discours (juridique, policier, déontologique, populaire, etc.) voudrait ou prétendrait le ménager en identifiant le coupable ou l'origine de la douleur. En insistant sur les écueils qui émanent de l'événement - fictif ou non — plutôt que sur l'origine 
de l'événement, le geste littéraire, au lieu de réparer un tort ou une blessure, défile et refile plutôt sa fidélité imparfaite et infiniment incomplète à la réalité : «Le roman, au fond est perçu par les tenants de la véracité comme un plagiat de l'autobiographie. Comme si la fiction n'était jamais que la copie, ou le masque, d'un texte plus réel qui viendrait d'un je certifié d'origine. " (Darrieussecq, 2010: 17.) Tout semble effectivement indiquer que l'infamie de Foucault éclaircit ce qui lie le réel à la réalité en rendant caducs les « vieux mots d'ordre » (Darrieussecq, 2010: 17) de l'authenticité « et son pendant idéologique : l'indicible » (17). Le geste de Darrieussecq a peut-être été calomnieux, mais peu ont pris le temps de montrer que l'on fait silence sur quelqu'un par pudeur ou bien par crainte de sa force, de son danger, et non de sa faiblesse.

Plutôt que se confronter à des exceptions théoriques ou historiques dont le but serait de montrer qu'il y a une causalité entre l'infamie et la diffamation, Foucault admet ouvertement la valeur négative de son entreprise herméneutique. II refuse de se ranger du côté de la victime « quelle que soit l'abjection que suscite le pouvoir qui l'écrase; c'est comme s'il nous conseillait d'être prudents, car cela fait partie du mécanisme de cette machine à représenter que d'attirer le regard du côté de celui qui contrevient et succombe » (Colucci, 2005: 144). Selon toute vraisemblance, Darrieussecq sollicite une parole révoltée - dans le cas de Rapport de police (2010) en puisant dans le bassin du genre de l'essai - pour construire une tactique de résistance sérielle. La série, comme l'entend Martine Delvaux dans Les filles en série, consiste à

remplacer l'identité par les alliances et les alliages, les contagions, les épidémies, le vent. Remplacer le nom propre par un ensemble à la troisième personne qui rapporte l'énoncé non plus à un sujet d'énonciation mais à un agencement collectif, qui destitue tout sujet au profit d'un agencement. (Delvaux, 2013: 28.)

En travaillant sur le dispositif de la sérialité pour penser le pouvoir des filles en série, Delvaux précise : « les filles en série refusent l'immobilité. Elles refusent l'essentialisme, la loi de l'identitaire. Elles manœuvrent, proposent de fausses pistes, séduisent pour prendre au piège [...]. Elles incarnent l'Ingourvernable. » (213.) C'est, croyons-nous, pour cette raison qu'il convient de lire quelques-unes des œuvres à l'étude avec cette infamie foucaldienne en tête. II n'est guère besoin d'insister sur l'extraordinaire essor des accusations qui traversent l'histoire française du début du XIX ${ }^{\mathrm{e}}$ jusqu'au XXI ${ }^{\mathrm{e}}$ siècle, que les études de Sapiro (2011), Tricoire (2011) et Compagnon (2017) ont mis de l'avant. Nous nous intéressons ainsi moins, dans cet article, aux relations tendues entre le littéraire et le juridique dans l'espace social durant les années 2000 qu'à la façon dont les mécanismes spécifiquement littéraires reconfigurent les effets du discours.

\section{Partir à l'assaut contre l'hygiène familiale}

Contrairement à Darrieussecq, Christine Angot fait l'objet de plusieurs poursuites judiciaires (diffamation ou 
atteinte à la vie privée) et d'accusations publiques (pour propos calomnieux ou infamants). Le Marché des amants (2008) avait valu à l'auteure une réception critique remplie de «foudres et moqueries » (Sulser, 2011). C'est au sein de ce roman qu'Angot avait mis en scène Élise Bidoit et deux de ses enfants sous leurs vrais prénoms d'origine éthiopienne (Kebra et Tafari), rendant ainsi le contrat de lecture (suspension de l'incrédulité) difficilement envisageable — en dépit du fait que les œuvres d'Angot ont, dès la parution de L'Inceste (1999), été associées à la forme autofictionnelle (Schaal, 2017; Baillargeon, 2014; Payot, 2011; Bridet, 2004). Angot a accepté de dédommager la mère à hauteur de 10000 euros sous la menace d'une procédure judiciaire. Mais Angot ne s'en est pas tenue là. Elle semble avoir repris le thème de la rupture amoureuse dans Les Petits (2011), cette fois-ci en mettant davantage en avant la relation entre Billy et Élise. Le 27 mai 2013, la 19 Chambre du tribunal de grande instance (celle qui est spécialisée dans les affaires de presse et d'édition) la trouve coupable pour atteinte à l'intimité de la vie privée (diffamation) et Angot doit verser, avec sa maison d'édition Flammarion, 40000 euros à Élise Bidoit. Cette dernière se serait reconnue dans le personnage d'Hélène Lucas, une mère de plusieurs enfants ayant vécu une rupture conjugale avec Billy, d'origine antillaise $\underline{5}$.

Avec Les Petits (2011), Angot reprend l'histoire familiale de Bruno en déplaçant l'accent du trio Bruno (nommé aussi doc Gynéco dans le livre), Christine et sa fille Léonore - mis en place dans Le Marché des amants (2008) - vers un autre trio, celui de Billy, Hélène et leurs enfants. Angot « s'infiltr[e] [de nouveau] dans le microclimat du premier bonheur », celui d'une famille réelle « assez ordinaire » (Rérolle, 2011) : Charly Clovis, un père antillais, musicien, et Élise Bidoit, une femme sans profession, qui devient une mère au foyer. Mais cette réécriture est moins un simple geste de répétition identique qu'un travail « riche en occurrences cultivées » où les romans «viennent former tresse » (Dubois, 2011: §22). Dans le cas plus spécifique de Christine Angot, nous posons plus particulièrement l'hypothèse que l'infamie tiendrait à des mécanismes littéraires spécifiques (intertextualité, réécriture, allusion, déjà-vu). Elle dérange parce qu'elle est une «bâtarde libre », selon la formule de Thierry Guichard (1997: 18). Pour Francesca Forcolin, Angot

place son écriture sous le signe de la transgression, de l'insolence. [...] La volonté est de bouleverser ce qu'il faut dire, ce que la société nous a imposé de dire, [...] [d']aller contre le bien-dit, les speech codes, contre, on pourrait dire, l'hypocrisie sociale ou la cosmétologie verbale - comme dirait Umberto Eco, qui voit dans le « politiquement correct » une solution outrageuse qui change le nom mais ne résout pas le problème. (Forcolin, 2011: 52.)

Chez Angot, la transgression semble déranger car, comme chez Darrieussecq, elle reprend, elle ressasse « ce qui fait mal » (Rérolle, 2010). Citant Philippe Forest, l'on peut dire donc que le geste littéraire entretient un rapport « avec l'expérience et avec la vérité » et qu'il prend « la forme d'une obsession » (Latour, 2008: 184) : 
Angot, comme Darrieussecq, revient livre après livre sur ce qui a déjà été écrit, mais qui ne devrait pas être dit de cette façon-là. Pour paraphraser Forest (198), disons qu'elles reviennent sur le refus d'en finir avec la distinction entre réel et réalité.

La question de l'intertextualité au sein de l'œuvre d'Angot a déjà été étudiée comme ouverture à l'énonciation d'un sujet non monolithique. Pour Forcolin, Angot veut « mettre en évidence la logique incestueuse de son écriture » (2011:55) afin de mettre « mal à l'aise le lecteur » (56) et de produire une « confusion vertigineuse » (56). David Ruffel considère que l'intertextualité angotienne s'inscrit « à la faveur d'une énonciation résolument plurielle » (Ruffel [2000] cité par Baillargeon, 2010: 60), la question du « mineur » traduisant alors une structure qui déterritorialise les schèmes œdipiens de la « fixité identitaire » (Baillargeon, 2010: 7). Telle une série autofictionnelle, des parties d'histoires qui étaient présentes dans Le Marché des amants (2008) sont réinscrites dans Les Petits (2011), avec cependant un changement paradigmatique de premier ordre. Le premier roman raconte l'histoire du point de vue de Christine Angot. Le second est le récit à propos d'une mère blanche et bourgeoise qui prend en otage ses petits contre leur père et, en retour, du père qui se bat pour les voir. Le récit place deux anciennes intrigues (la rupture entre Hélène et Bruno/Billy et la rencontre entre Christine et Bruno/Billy) dans un monde déjà conceptualisé, déjà matérialisé. Cependant, il y a un effet de démultiplication réalité-réel et de dédoublement entre ces deux romans. Reprenant à notre compte l'analyse de Philippe Forest, l'on peut dire que l'infamie qui se donne à lire dans l'écriture angotienne n'a pas tant à voir avec les thèmes de la rupture familiale et de la rencontre amoureuse qu'avec les restes qui demeurent quand le réel et la réalité de ces événements se rencontrent:

Le « réel » — en tant qu'il a partie liée avec l'impossible — n'est un « thème » que dans la mesure très exacte où ce thème a le statut d'un reste. Et à ce dernier mot, pour éclairer son sens, on peut rendre d'abord son acception mathématique. Divisez 10 par 3 : aussi loin que vous puissiez au-delà de la virgule, quelque chose vous demeure du dividende, et ce « quelque chose » laisse tout calcul inachevé. [...] Ce « quelque chose » est là qui persiste, hétérogène par rapport à l'ordre du discours, ne se laissant pas dissoudre à l'intérieur de lui, et appelant du coup l'écho d'une parole — le roman — qui saura le recueillir, ce reste dont la « réalité » ne veut pas, où il n’a pas sa place. (Forest, 2007: 47.)

Avec Les Petits (2011), les noms, les êtres, les lieux, ne sont pas des substituts pour une matérialité empirique (le vrai nom, le vrai lieu), mais ouvrent sur une dialectique de signes qui dérangent parce qu'ils ne sont pas censés revenir. Les reprises et les prolongements des personnages (de deux enfants à cinq, de Bruno à Billy, mais supposément la même Hélène et la même Christine), des lieux (de Paris à la Martinique), portent à croire que ces romans s'envahissent l'un l'autre. Au lieu de clarifier les enjeux qui y sont rappelés, le jeu des rapprochements intensifie la confusion entre le texte premier et le texte second, « dont on dirait volontiers que 
l'écriture est source d'une dépossession mutuelle, chacun des récits parlant pour l'autre » (Dubois, 2011: §22). Le tout petit lien qui reste après que le dénominateur (réel) et le numérateur (réalité) sont divisés a sans doute quelque chose à voir avec l'infamie, car celle-ci montre le réel qui languit formant toute une tératologie de filiations. Telle une constellation, ces filiations se télescopent et s'enchevêtrent entre les plis du réel et de la réalité si bien que toute forme de « trivialité qui dominer[ait] le roman se renverse en parole [...] imposante » (Dubois, 2011: §22) du passé. Celle-ci vient hanter le social tout en refusant simultanément de garder ce «non-dit », cette « horreur » (Forcolin, 2011: 58) invisible ou indicible.

Par ailleurs, si au début des Petits (2011) il n'y a pas de « confusion vertigineuse » (Forcolin: 56), il n'en va pas de même vers le milieu du roman, où la subjectivité du « je » (Christine Angot) expose de la façon « la plus détaillée [...] son expérience [sexuelle] cauchemardesque » (Havercroft, 2012: 161). Jusqu'au milieu du roman, Angot est décrite à la troisième personne et la narration en parle au même titre et au même niveau que les autres personnages. Elle n'est que l'amoureuse de Billy et la belle-mère en devenir des enfants de Billy. En effet, lors de la première lecture des Petits, il n'y a pas de « je-Christine Angot » à l'horizon jusqu'à ce que la machine de la vérité se dérègle au milieu du récit :

II s'assoit en face de moi. II me demande si je sais ce qu'il a à me dire. Si je ressens la même chose que lui, ou si j'étais à mille lieues d'imaginer. On s'embrasse. Puis on fait l'amour. (Angot, 2011: 116.)

Cette incursion du « je » apporte une autre dimension symbolique à la structure familiale. L'écriture s'attarde ainsi sur l'inquiétante relation de l'autre figure de la mère pas tout à fait mère se posant en témoin ternaire face au couple parental rompu :

Je reste à l'écart. Je ne sais pas si je dois rester près de Billy ou loin. Je me sens en faute. Jugée.

Maladroite. Je ne trouve pas de bonne attitude pour mon corps [...]. C'est bête. Justement je me sens bête. Illégitime. Inutile. Nulle. Sans motif pour être là. Sans droit. Sans titre. J'ai l'impression d'être un parasite. Une molécule inutile. (Angot, 2011: 122.)

La présence des phrases nominales ou verbales saccadées, la répétition des locutions adverbiales de la négation, l'utilisation de métaphores de la bêtise (« maladroit », " bête ») ainsi que l'insertion du registre de la pathologie virale aident le lectorat à mieux comprendre comment la figure de l'autre mère ajoute une préoccupation symbolique au « je " similaire à celle dont parle Mary Douglas à propos de la souillure du tabou : la pollution « est un système symbolique qui repose sur la représentation du corps et dont le but essentiel est d'ordonner une hiérarchie sociale » (2001 [1967]: 140). Les phrases courtes sont communes chez Angot et, selon l'analyse d'Arnaud Jacob, elles « émanent d'un corps dont elles ne peuvent être coupées, d'un corps qui est dit ou suggéré, affleure [sic] partout à la surface de l'écrit » (Jacob [2008] cité par Baillargeon, 2014: 43). 
Aussi est-il question ici d'une présence qui ne se donne pas seulement à lire à la surface, mais comme « un parasite [,] une molécule inutile » (Angot, 2011: 122). Le « je » Angot vient infecter le corps familial d'un virus étranger. Angot n'est pas seulement un sujet au même titre que les autres personnages : elle est un intrus « illégitime » (122) de la famille nucléaire, la souillure qui vient infecter l'hygiène familiale.

On voit ainsi comment l'apparition du « je-Christine » dans l'extrait cité plus haut dérègle la propreté du corps familial, lequel ne peut plus être contenu. « Je-Christine » fait obstacle à une scène qui, au demeurant, devrait rester invisible du regard public, propre de toute souillure causée par un contact avec un corps étranger. La sentence juridique autour des Petits (2011) a essentiellement porté sur l'atteinte à la vie privée (Edwards, 2018). Mais on peut lire dans le discours médiatique que c'est spécifiquement l'incursion de la belle-mère dans la vie des enfants qui n'a pas conféré un sentiment de protection chez la mère Élise Bidoit (Sulser, 2011). Lors de l'audience, la plaignante, dont les propos sont cités dans Le Nouvel Observateur et repris dans Le Temps, a déclaré : « Je voudrais qu'on comprenne ma blessure et ma souffrance. L'enjeu de ce livre ce sont les petits. Et les petits, ce sont mes enfants. » (Bidoit citée par Sulser, 2013.) Pour l'avocat, Me William Bourdon, la somme de 40000 euros à verser par Christine Angot et son éditeur Flammarion à Élise Bidoit était insuffisante en regard de l'acharnement et de la menace d'une femme qui accueille ses enfants, chez elle (Sulser, 2011). Comme le signale l'étude de Peter Gossage sur l'imaginaire de la belle-mère en Occident, " parmi tous les archétypes issus du milieu domestique, la société a toujours été le plus méfiante de la belle-mère $\underline{6}$ » (1995: 564). Que ce soit en France, au Québec, ou ailleurs, « dans les proverbes, les contes de fées, ou dans d'autres formes de la culture populaire ", la belle-mère continue de représenter « les idéologies dominantes de la maternité et de la vie familiale $\underline{z}$ » (565). Car c'est la famille nucléaire, les rapports familiaux bourgeois ou la notion essentialiste de maternité, entre autres, qui sont encore élevés au rang d'idéaux a priori. Quoiqu'elle ne sache pas où ni comment poser son corps et son regard, Angot reste là, elle habite la maladresse de la bellemère et, ce faisant, elle refuse son rôle de tierce exclue de la scène familiale. Ce rôle d'intrus lui permet de se rapprocher d'une scène qui ne lui appartient pas. Ce rôle révèle jusqu'à son ultime point de saturation la nature du besoin, considéré inquisiteur et impudique, de connaître les secrets de la famille des autres, largement « à la base de nombre de controverses, dont l'autofiction est la victime fréquente » (Viart, 2009: 106). Angot déplie l'arraisonnement des structures familiales auquel certaines pratiques institutionnelles et individuelles donnent lieu, notamment, lorsqu'elles traitent d'intrus ceux qui ne cadrent pas bien avec la norme.

\section{Le bruissement de la violence}

Angot n'est pas seulement un intrus de la famille nucléaire. Elle s'attarde aussi à la figure du père dansLes Petits (2011). Elle utilise cette place illégitime de l'intrus pour déplier le « clivage politique imposé » du Noir dans le logos de la famille bourgeoise (Joos, 2000: 97). Pis encore, elle met en lumière les effets néfastes des 
règlements instaurés par Hélène au sein de la famille. Billy « comprend et il est d'accord » avec ces règlements (Angot, 2011: 42). Même s'il semble consentir ou acquiescer aux prescriptions maternelles, le roman insiste sur un fait : “ il y a un excès dans l'application. Un côté doctrinaire, un manque d'air. Hélène exerce son pouvoir règlementaire par l'hygiène. » (42.) Ce pouvoir sur le monde s'étend à petits coups, « des petites règles, des petits trucs, des petites restrictions " (42), si bien que ce monde se rétrécit à mesure que les enfants grandissent. «[C]ertains détails se modifient », Hélène « assouplit leur régime alimentaire, et réintroduit les œufs » (42), mais « [l]e territoire est surveillé » (42) dans la totalité du roman, même quand Billy n'a plus la garde des enfants. La mère « est la pointe du compas » (47), la créatrice de « ses propres lois, [...] le rayon d'action » qui « touche tout ce qui a un rapport avec elle » (47). Hélène est partout, dans la loi juridique comme dans la loi familiale. Elle devrait être en droit de déterminer ce qui déroge à l'ordre puisqu'en elle et par elle émanent les dichotomies essentielles à l'hygiène familiale : «Billy travaille mais n'est pas absent de la maison » et pourtant : «Rien d'autre ne compte vraiment. Rien d'autre que la propreté. » (46.) Le texte expose les couches souterraines du discours : celles de la violence des petites choses qui figurent comme autant de signes attestant des modes de surveillance et d'emprisonnement servant à limiter, voire à exclure, non pas tant le père du cercle familial, mais le Noir de la société française.

Par ailleurs, les personnages minoritaires sont présentés comme des instigateurs de l'économie raciale et raciste. Un jour, Billy se rend au Monoprix pour faire l'épicerie, dont les achats respectent le nouvel « assouplissement » (Angot, 2011: 47) nutritionnel d'Hélène. Tenant les sacs d'épicerie dans ses mains, Billy est sur le point de sortir du supermarché lorsque « une femme blonde, sans âge, détale à toutes jambes devant lui » (47). Billy rentre « à la maison exaspéré, en colère contre cette société malade, raciste » (48). C'est la violence du petit geste de racisme pas si anodin de la femme blanche et indistincte, transparente et pourtant si puissante dans sa capacité de l'accaparer, qui s'impose à Billy.

Angot avait déjà abordé la violence du racisme dans le Marché des amants, mais autrement. Y contrastent Bruno Beausir, un métis issu de la banlieue, et Marc, un homme blanc issu d'un milieu intellectuel. Alice Granger note : « il y a ce préjugé selon lequel le milieu défavorisé de Bruno, celui d'où il vient, doit être réparé, le milieu favorisé, ici le milieu littéraire et intellectuel, étant la référence. » (2008.) II est important de mentionner que ce roman ne montre pas seulement l'opposition entre les hommes, Bruno et Marc. Car Christine renforce l'exclusion de Bruno. Elle peut faire sortir Bruno de son milieu; c'est elle qui possède le capital (culturel, symbolique, économique) nécessaire à l'émancipation de Bruno. L'on pourrait même dire que l'expérience de Billy dans Les Petits est « couplée » (Dubois, 2011: §8) à celle de Bruno dans Le Marché des amants, dont le titre « évoque une phrase prononcée [par le père d'Angot] : sur le marché des amants un Noir vaut moins qu'un Blanc » (Granger, 2008). Angot ne répète pas la même scène de violence entre la séquence au Monoprix dans Les Petits et la violence racisée dans Le Marché des amants. Elle fait néanmoins languir la 
même violence du petit geste pas si anodin. Ce qui reste entre les deux textes répond peut-être à un impératif de relancer dans l'avenir de l'écriture la possibilité de comprendre les fonctionnements de ces petits gestes de violence dont le bruissement émerge comme un contre-courant lequel ne finit pas de se faire sentir.

Cette forme de réécriture ouvre une nouvelle configuration du livre à venir. Elle laisse entrevoir l'insistance d'un refus de l'auto-pardon. Cet appel que chaque livre adresse à l'avenir, un passé déjà advenu quand les livres sont déjà là, témoigne d'une incompréhension ressentie face à un événement, certes intelligible, mais dont le réel lève le voile sur la secousse. Et d'une certaine manière, avec la réécriture sous forme de série autofictionnelle, Angot réinscrit une vision aveuglante de sa propre cécité face aux violences qu'elle commet. Elle n'est pas la bonne femme blanche. Elle n'est pas hors des lois. Entre ces deux romans, Angot ressemble à la « femme blonde, sans âge » (2011: 47). Comme ce personnage secondaire dans Les Petits, dans Le Marché des amants, Christine « est [...] saisie de peur, de très grande inquiétude » (Granger, 2008) face à Bruno. Granger a raison de préciser :

Ce que ce roman met de manière brillante à la lumière, c'est la résistance exceptionnelle de Bruno à cett€ sorte d'insémination culturelle cherchant à réparer un tiers exclu selon un critère d'appartenance. [...] Bruno en a, comme on dit, il sait se battre même dans ces zones de non-droit où il a grandi et a appris à déjouer les dangers infinis. (Granger, 2008.)

Le geste de réécriture chez Angot ne consiste donc pas à « remodeler la vie d'autrefois » (Lis, 2008: 202). Au lieu de tenter d'annuler les excrétions et exceptions - pour reprendre ici le vocabulaire de la souillure du tabou — qui perturbent l'ordonnancement du discours ou de tenir l'objet de la répulsion en marge du monde, ce travail de l'infamie, insiste, revient, renaît et le geste littéraire semble être le lieu qui illustre, non pas le résultat du contrôle de ces expulsions, mais la dynamique circulaire qui lie les évacuations aux processus de filtration révélés par les restes du discours, restes que Foucault compare à des « particules dotées d'une énergie d'autant plus grande qu'elles sont elles-mêmes plus petites et difficiles à discerner » (1977: 12).

\section{Les tout petits qui restent}

Dire l'indicible, montrer le non-dit, dévoiler l'horreur et la violence cachées au sein de la société, ressemble à un geste qui n'est pas seulement réservé à la narration autobiographique, autofictionnelle ou fictionnelle. Si les œuvres d'Angot sont infâmes, c'est que l'auteure ne « RACONTE pas » : « Je ne raconte pas MON histoire. Je ne raconte pas une HISTOIRE. Je ne débrouille pas MON affaire. Je ne lave pas MON linge sale. Mais le drap social. 》 (Angot, 2000: 161-162.) Angot ne se sert pas de son écriture pour s'excuser ou se disculper de ses gestes déplacés, inacceptables ou racistes. Elle risque son existence pour rendre cette écriture publique afin que le réel se fasse « pli du drap social dans lequel d'autres plis viennent se déplier et se révéler (Angot 
affectionne ce mot de pli pour parler de la société) » (Dubois, 2011: §19). Tout le travail d'Angot consiste donc à se faufiler dans les plis de la vie, du tissu social, pour révéler que « ce qui est scandaleux, c'est la distance ou l'étrangeté entre ce que nous croyons et ce qui, à notre insu, agit » (Foucault, 1977: 23-24). L'aspect dédoublé dans les deux romans de la rencontre avec l'amant antillais, du dédoublement Bruno/Billy et de celui de ses enfants, ne sert pas tant à précariser la santé ou le caractère prétendument sacré du nœud familial nucléaire des Clovis-Bidoit. Angot ne s'acharne pas - comme le pensent Bidoit ou son juriste - à ternir le visage de la mère biologique. Elle garantit au réel un effet de déjà-vu. Le déjà-vu littéraire n'est pas tant « une reprise parfaite de quelque chose qui vient à la place du présent » qu'un don d'« intensité » (Mavrikakis, 2011: 67), une force, une puissance de la récidive. Angot fait autant de ses œuvres une œuvre infâme qu'elle dévoile l'objet mystérieux de la véritable infamie : les vies ordinaires.

À la question posée au début de cet article, une interrogation autour de la distance appropriée duproche, l'on peut répondre qu'Angot est paradoxalement celle qui raconte sa vie et « dont la subjectivité est envahissante » (Dubois, 2011: §23) pour les autres vies qu'elle côtoie. Le « je » de sa propre vie est aussi la tierce personne de la vie des autres : une Autre hors-mariage, ex-filio, une amante et une marâtre. Son geste littéraire amplifie ainsi la force de cette figure de mère de remplacement, tout comme il rend visible l'invisibilité du Noir dans l'inconscient social- . Ce regard ne trouble pas tant la distribution des forces du pouvoir dans nos sociétés qu'il cristallise le profond sentiment de malaise que la société entretient envers, d'une part, le « Noir », symbole d'un tabou, toujours prêt à déborder, à frapper, à démolir l'hygiène de la société et, d'autre part, de la marâtre, laquelle « [d]ans l'imaginaire populaire [...] est souvent décrite comme séductrice, égoïste, vaniteuse, avide, vengeresse, mensongère, malveillante, manipulatrice, indigne de confiance, toute-puissante et redoutable » (Laflamme, 2002: 107).

Depuis les années 2000, le geste de la récidive caractéristique des séries autofictionnelles chez Angot et Darrieussecq engage une réflexion allant au-delà du devoir éthique, lequel consisterait à privilégier les souffrances actuelles ou empiriques, mais incompréhensibles. Angot avait déjà déclaré après la parution du roman L'Inceste, lors de l'émission Tout le monde en parle: “Ce qu'il y a à écrire, c'est justement ce que les gens ne peuvent pas comprendre. " (Ardisson et Hardy, 1999: 67 min -67 min 10 s.) Les œuvres ici étudiées posent et reposent ainsi la même question : sommes-nous bêtes, bêtes comme Christine devant la scène familiale étrangère, aussi anodine soit-elle? Dans un entretien avec Yoann Moreau, l'anthropologue français François Laplantine le dit bien à propos du langage artistique des catastrophes ordinaires : prenant en exemple Clarice Lispector pour parler du registre littéraire de l'intime, il affirme que l'art « parvient à traduire par de tout petits mots, des détails et des modulations infimes, des catastrophes amoureuses, des déceptions 》 (2015: 29). Angot et Darrieussecq ne sont pas des exceptions à ce que peut faire la littérature. Les petits inscrits et retravaillés au sein de leurs textes sont certes des matériaux inquiétant l'ordre du discours. Néanmoins, ils sont 
ce avec quoi le geste littéraire se démène et travaille à déplier le monde, comme si la constance même de ces récidives n'était autre chose qu'une résistance plus insistante d'un réel qui refuse de se ranger du côté des bonnes intentions.

1. «Un certain nombre de femmes écrivains [Christine Angot, Marie Darrieussecq, Virginie Despentes et Catherine Millet] publient depuis quelques années des récits rencontrant un réel succès et laissant une large place à la sexualité et, plus largement, au corps. Les scandales et leurs relais dans la presse, ainsi que les chiffres de vente élevés qui les accompagnent, se succèdent régulièrement depuis la fin de l'année 1994. ”(Bridet, 2004: 440.)

2. L'article de la loi du 29 juillet 1881, signé par le Président de la République Jules Grévy, a été modifié par ordonnance du 6 mai 1944 (art. 4). On peut y lire désormais la loi du 29 juillet 1881 sur la liberté de la presse dans la version consolidée au 8 mai 2019 : " Toute allégation ou imputation d'un fait qui porte atteinte à l'honneur ou à la considération de la personne ou du corps auquel le fait est imputé est une diffamation. La publication directe ou par voie de reproduction de cette allégation ou de cette imputation est punissable, même si elle est faite sous forme dubitative ou si elle vise une personne ou un corps non expressément nommés, mais dont l'identification est rendue possible par les termes des discours, cris, menaces, écrits ou imprimés, placards ou affiches incriminés. Toute expression outrageante, termes de mépris ou invective qui ne renferme l'imputation d'aucun fait est une injure. » (FRANCE, 1944 [1881].) La version à laquelle Antoine Compagnon se réfère est celle datant de 1881 et non celle de 1944.

3. Voir à ce propos le chapitre 6 de son Rapport de police (Darrieussecq, 2010: 141-177).

4. L'article de Maïté Snauwaert dans le présent dossier thématique, sous l'intitulé « D'une infamie l'autre », étudie de près la polémique opposant Laurens et Darrieussecq autour de Tom est mort (Snauwaert, 2019).

5. Pour une analyse en profondeur des procédures juridiques chez Christine Angot, prière de lire Natalie Edwards (2018).

6. [Je traduis.]

7. [Je traduis.] Peter Gossage cite les travaux de David Noy (1991), de Peter Laslett (1973), de Robert Darnton (1985) et de Natalie Zemon Davis (1987). Je note par ailleurs les études d'Hélène Deutsch (1969 [1945]), de Valérie Laflamme et Hélène David (2002) et de Sylvie Perrier (2006).

8. Le terme « inconscient social » est issu, entre autres, des travaux de Pierre Bourdieu. Sous la question de l'usage sociologique du concept d'inconscient, il existerait pour Bourdieu un rapport fort entre les structures mentales (psychiques) et sociales (individuelles, collectives, institutionnelles) sans que l'inconscient soit automatiquement un opérateur de finalité, c'est-àdire, sans que l'inconscient soit une détermination psychique détachée du social. Pour Bourdieu, l'inconscient social implique qu'il y a des habitus : " des systèmes de dispositions durables et transportables, structures structurées prédisposées à fonctionner comme structures structurantes, c'est-à-dire en tant que principes générateurs et organisateurs de pratiques et de représentations qui peuvent être objectivement adaptées à leur but sans supposer de visée consciente de fins et la maîtrise expresse des opérations nécessaires pour les atteindre, objectivement et sans être en rien le produit de l'obéissance à des règles, et étant tout cela collectivement orchestrées sans être le produit de l'action organisatrices d'un chef d'orchestre. » (1980: 120-121.)

\section{Bibliographie}

Angot, Christine. 1999. L'Inceste. Paris : Stock, 216 p.

Angot, Christine. 2000. Quitter la ville. Paris : Stock, 204 p.

Angot, Christine. 2008. Le Marché des amants. Paris : Seuil, «Fictions \& Cie », 324 p.

Angot, Christine. 2011. Les Petits. Paris : Flammarion, 196 p.

Ardisson, Thierry et Linda HARDY. 1999. «Entrevue avec Dieudonné, Christine Angot, Clémentine Célarié et David Halliday », dans Tout le monde en parle. France 2, Disponible dans les archives de l'INA diffusées sur Youtube, $65 \min 20 \mathrm{~s}$ à $86 \min 7 \mathrm{~s} \min .<$ https:/www.youtube.com/watch?v=rWVGDHWv7Jk>.

Consultée le 14 mai 2019. 
BAILLARGEON, Mercédès. 2010. « «e personnel est politique. » La figure de l'inceste dans l'œuvre de

Christine Angot ». Mémoire de maîtrise. Département d'études littéraires, Université du Québec à

Montréal, 106 f. <https://core.ac.uk/download/pdf/9539264.pdf>. Consultée le 27 mai 2019.

BAILLARGEON, Mercédès. 2014. «Esthétique et politique dans l'autofiction des femmes contemporaines».

Thèse de doctorat. University of North Carolina at Chapel Hill, $202 \mathrm{f}$.

Bourdieu, Pierre. 1980. Le sens pratique. Paris : Les Éditions de Minuit, 480 p.

BRIDET, Guillaume. 2004. «Le Corps à l'CEuvre des femmes écrivains. Autour de Christine Angot, Marie

Darrieussecq, Virginie Despentes et Catherine Millet », dans Bruno BLANCKEMAN, Alain Mural-Brunel et Marc Dambre (dir.), Le Roman français au tournant du XXIe siècle. Paris : Presses Sorbonne Nouvelle, p. $439-447$.

CAvIGLIOLI, David. 2013. «Les romanciers peuvent-ils encore s'inspirer de personnes réelles? ». Le Nouvel Observateur, 4 août. $\not$ https://bibliobs.nouvelobs.com/actualites/20130628.OBS5848/les-romancierspeuvent-ils-encore-s-inspirer-de-personnes-reelles.html>. Consultée le 14 mai 2019.

Coluccl, Mario. 2005. «Hystériques, internés, hommes infâmes. Michel Foucault et la résistance au pouvoir ». ERES, vol. 1, no 20, p. 123-145.

Compagnon, Antoine. 2017. «De la littérature comme sport de combat ». Cours au Collège de France Littérature française moderne et contemporaine. Histoire, critique, théorie, Paris, 3 janvier au 28 mars. Collège de France. <https://www.college-de-france.fr/site/antoine-compagnon/course-2017-03-0716h30.htm>. Consultée le 15 mai 2019.

DARnton, Robert. 1985. The Great Cat Massacre and Other Episodes in French Cultural History New York : Vintage Books, $320 \mathrm{p}$.

DarrieussecQ, Marie. 1998. Naissances de fantômes. Paris : P.O.L., 160 p.

DarRieussecQ, Marie. 2007. Tom est mort. Paris : P.O.L., 256 p.

DARRIEUSSECQ, Marie. 2010. Rapport de police. Accusations de plagiat et autres modes de surveillance de la fiction. Paris : P.O.L., 319 p.

Delvaux, Martine. 2013. Les filles en série. Des Barbies aux Pussy Riot Montréal : Remue-Ménage, 280 p.

DemArtinI, Anne-Emmanuelle. 2002. «L'infamie comme œuvre. L'autobiographie du criminel Pierre-

François Lacenaire ». Société \& Représentations, no 13, avril, p. 121-136.

DeutSch, Hélène. 1969 [1945]. «Les belles-mères », dans Hélène Deutsch (dir.), La psychologie des femmes.

Paris : Presses Universitaires de France, t. 2, p. 373-390.

Douglas, Mary. 2001 [1967]. De la souillure. Essai sur les notions de pollution et de tabou traduit par Anne Guérin. Paris : La Découverte, 210 p.

Dubols, Jacques. 2011. «Christine Angot. L'enjeu du hors-jeu». COnTEXTES: revue de sociologie de la littérature, no 9, 1er septembre. <https://journals.openedition.org/contextes/4789>. Consultée le 14 mai 
2019.

EdwARDS, Natalie. 2018. «Autofiction and the Law. Legal Scandals in Contemporary French Literature». Contemporary French and Francophone Studies: Sites, vol. 22, no 1, p. 6-14.

FANTHOU, Bastien. 2008. «Les attentats à la pudeur dans la Corrèze de la Belle Époque». Siècles. Cahiers du Centre d'histoire «Espaces et Cultures», no 28, p. 11-23.

ForColIN, Francesca. 2011. «Christine Angot: Le désir d'indigner le lecteur. La société violée par l'(auto)fiction ». Carnets. Revue électronique d'études françaises de l'APEF, vol. 1, no 3, p. 51-61. $<$ https://journals.openedition.org/carnets/6374>.

FOREST, Philippe. 2007. Le Roman, le réel et autres essais. Allaphbed volume 3 Nantes : Éditions Cécile Dufaut, $302 \mathrm{p}$.

Foucault, Michel. 1977. «La vie des hommes infâmes ». Les Cahiers du chemin, no 29, p. 12-29.

FRANCE. 1944 [1881]. «Loi du 29 juillet 1881 sur la liberté de la presse». Legifrance.gouv.fr. France :

République française, 25 mars 2019. <https://www.legifrance.gouv.fr/affichTexte.do? cidTexte=LEGITEXT000006070722>. Consultée le 8 mai 2019.

Gossage, Peter. 1995. « La marâtre. Marie-Anne Houde and the Myth of the Wicked Stepmother in Quebec ». The Canadian Historical Review, vol. 76, no 4, p. 563-597.

Granger, Alice. 2008. “"Le Marché des amants", Christine Angot ». e-litterature.net, 17 octobre. $<$ http://www.e-litterature.net/publier2/spip/spip.php?page=article5\&id article=658 $>$. Consultée le 14 mai 2019.

GuiCHARD, Thierry. 1997. «Christine Angot, la bâtarde libre ». Le matricule des anges, no 21, p. 18-19. HAVERCROFT, Barbara. 2012. «Splendeurs et misères de la confession au féminin au XXle siècle», dans Bruno Blanckeman et Barbara Havercroft (dir.), Narrations d'un nouveau siècle. Romans et récits français (2001-2010), colloque de Cerisy. Paris : Presses Sorbonne Nouvelle, p. 159-172. <http://books.openedition.org/psn/482>. Consultée le 14 mai 2019.

Jess-Cooke, Carolyn et Constantine Verevis (dir.). 2010. Second Takes. Critical Approaches to the Film Sequel. New York : SUNY Press, $263 \mathrm{p}$.

Joos, Jean Erbest. 2000. «Clivage relationnel et violence. Éléments pour une théorie politique de la relation à partir de la pensée de Franz Fanon ». Fictions et politique, no 63, p. 96-108.

KAPRIĖLIAN, Nelly. 2010. «Marie Darrieussecq. Entretien avec Nelly Kaprièlian», dans Écrire, écrire, pourquoi? Marie Darrieussecq. Paris : Éditions de la Bibliothèque publique d'information, p. 3-23. <https://books.openedition.org/bibpompidou/1136>. Consultée le 14 mai 2019.

LAFLAMme, Valérie et Hélène DAVID. 2002. «La femme a-mère. Maternité psychique de la marâtre ». Revue française de psychanalyse, vol. 66, no 1, p. 103-118.

LAPLANTINE, François. 2015. «La dimension subie ». Communications, vol. 1, no 96, p. 19-38. 
LASLETt, Peter. 1973 [1965]. The World We Have Lost. England before the Industrial Age 2e édition revue. New York : Charles Scribner's Sons, $325 \mathrm{p}$.

LATOUR, Marie-José. 2008. «Entretien avec Philippe Forest ». ERES, vol. 2, no 11, p. 181-200.

Laurens, Camille. 2004 [1995]. Philippe. Paris : P.O.L., 75 p.

LaURens, Camille. 2007. «Marie Darrieussecq ou le syndrome du coucou ». La Revue littéraire, no 32, p. 114.

LIS, Jerzy. 2008. «Annie Ernaux et Philippe Vilain. Réécriture croisée», dans Magdalena WANDZIOCH (dir.),

Quelques aspects de la réécriture Katowice (Pologne) : Uniwersytetu Śląskiego W Katowicach, p. 202-213.

MAVRIKAKIS, Catherine. 2011. «Les "blind dates" de l'écriture. Rencontres durassiennes du présent ».

Dalhousie French Studies, vol. 95, été, p. 65-72.

NDIAYE, Marie. 1994. Un temps de saison. Paris : Éditions de Minuit, $144 \mathrm{p}$.

NDIAYE, Marie. 1996. La Sorcière. Paris : Éditions de Minuit, 192 p.

Noy, David. 1991. «Wicked Stepmothers in Roman Society and Imagination». Journal of Family History, vol. 16, no 4, p. 345-361.

Otchakovsky-Laurens, Paul. 2007. « Non, Marie Darrieussecq n'a pas "piraté" Camille Laurens ». Le Monde des livres, 31 août. «http://www.lemonde.fr/livres/article/2007/08/30/non-marie-darrieussecq-n-a-pas-piratecamille-laurens-par-paul-otchakovsky-laurens 949150 3260.html>.

РАYOT, Marianne. 2011. «La famille selon Christine Angot ». L'Express, 14 janvier. <https://www.lexpress.fr/culture/livre/les-petits 952291.html>. Consultée le 14 mai 2019.

Peker, Julia. 2010. Cet obscur objet du dégoût. Paris; Sofia : Le Bord de l'Eau, 194 p.

PerRIER, Sylvie. 2006. « La marâtre dans la France d'Ancien Régime. Intégration ou marginalité?». Annales de démographie historique, vol. 2, no 112, p. 171-188.

Rerolle, Raphaëlle. 2010. «"Rapport de police” de Marie Darrieussecq. Marie Darrieussecq publie un essai pour défendre son "honneur d'écrivain" ». Le Monde, 7 janvier. $<$ http://www.lemonde.fr/livres/article/2010/01/07/rapport-de-police-de-mariedarrieussecq_1288454_3260.html>. Consultée le 14 mai 2019.

Rerolle, Raphaëlle. 2011. «"Les Petits”, de Christine Angot. Deux femmes puissantes». Le Monde, 13 janvier. <http://www.lemonde.fr/livres/article/2011/01/13/les-petits-de-christine-angot_1464997_3260.html>. Consultée le 14 mai 2019.

Robert, Paul. 2008. Le Nouveau Petit Robert. Paris : Le Robert, 2837 p.

Roux, Joseph. 1882. Les délits de la Presse dans la loi du 29 juillet 1881, suivi d'un appendice sur le projet de loi concernant les publications contraintes aux mœurs voté le 27 juillet 1882. Amiens : Fousseau-Leroy, 218 p. <https://gallica.bnf.fr/ark:/12148/bpt6k5659671r.textelmage>. Consultée le 14 mai 2019. 
RuBy, Christian. 2011. «L’objet du scandale observé par une juriste». nonfiction.fr, 21 mars. $<$ https://www.nonfiction.fr/article-4408-lobjet-du-scandale-observe-par-une-juriste.htm>. Consultée le 14 mai 2019.

SAPIRO, Gisèle. 2011. La Responsabilité de l'écrivain. Littérature, droit et morale en France (XIXe-XXIe siècle). Paris : Seuil, $752 \mathrm{p}$.

ScHAAL, Michèle. 2017. Une troisième vague féministe. Les femmes de lettres de la nouvelle génération Leyde : Brill et Rodopi, $344 \mathrm{p}$.

Snaumaert, Maïté. 2019. «D’une infamie l'autre. La mort de l'enfant en régime de fiction». Captures, vol. 4, no 1 «Paroles diffamantes, images infamantes », mai. 丸ttp://www.revuecaptures.org/node/3317/>. Consultée le 29 mai 2019.

Strasser, Anne. 2012. «Camille Laurens, Marie Darrieussecq. Du "plagiat psychique" à la mise en questions de la démarche autobiographique ». COnTEXTES, no 10. $<$ http://journals.openedition.org/contextes/5016>.

SULSER, Éléonore. 2011. «Christine Angot dissèque l'échec d'une famille». Le Temps, 21 janvier. $<$ https://www.letemps.ch/culture/christine-angot-disseque-lechec-dune-famille-0>. Consultée le 14 mai 2019.

TricolRe, Agnès. 2011. Petit traité de la liberté de création. Paris : La Découverte, 304 p.

VIART, Dominique. 2009. «Le silence du père au principe du "récit de filiation" ». Études françaises, vol. 45, no 3, p. 95-112.

Wikipédia. [s. d.]. «Diffamation en droit français ». Wikipedia. <https://fr.wikipedia.org/wiki/Diffamation en droit fran\%C3\%A7ais>. Consultée le 27 mai 2019.

Wroblewski, Ania. 2016. La Vie des autres. Sophie Calle et Annie Ernaux, artistes hors-la-loi Montréal : Presses de l'Université de Montréal, $260 \mathrm{p}$.

Zemon Davis, Natalie. 1987. Fiction in the Archives, Pardon Tales and Their Tellers in Sixteenth-Century France. Stanford : Stanford University Press, $217 \mathrm{p}$. 